\title{
A Headache Diagnosis Method Using an Aggregate Operator
}

\author{
Jeong Yong Ahn ${ }^{1, a}$, Kyung Ho Choi ${ }^{b}$, Jeong Hyun Park ${ }^{c}$ \\ ${ }^{a}$ Department of Statistics (Institute of Applied Statistics), Chonbuk National University \\ ${ }^{b}$ Department of Basic Medical Science, Jeonju University \\ ${ }^{c}$ Department of Statistics, Chonbuk National University
}

\begin{abstract}
The fuzzy set framework has a number of properties that make it suitable to formulize uncertain information in medical diagnosis. This study introduces a fuzzy diagnostic method based on the interval-valued interview chart and the interval-valued intuitionistic fuzzy weighted arithmetic average(IIFWAA) operator. An issue in the use of the IIFWAA operator is to determine the weights. In this study, we propose the occurrence information of symptoms as the weights. An illustrative example is provided to demonstrate its practicality and effectiveness.
\end{abstract}

Keywords: Interval-valued fuzzy sets, fuzzy differential diagnosis, interview chart, IIFWAA operator, occurrence information of symptoms.

\section{Introduction}

It is widely accepted that the information available to the physician about a patient and about medical relationships in general is inherently uncertain (Adlassnig, 1986). Fuzzy set(FS) theory makes it possible to define the inexact medical information as fuzzy sets, therefore it can be utilized for modeling the diagnostic process.

An application of FS on medical science fields already proposed by Zadeh (1969) and Sanchez (1979) invented a fully developed relationships modelling theory of symptoms and diseases using FS. Since Atanassov (1986) introduced the concept of intuitionistic fuzzy set(IFS), FS theory has been used in the field of medicine. Later, Turksen (1986) introduced the concept of interval-valued fuzzy set(IVFS) and Atanassov and Gargov (1989) introduced the concept of interval-valued intuitionistic fuzzy set(IVIFS), which is a generalization of the IFS. The fundamental characteristic of the IVFS and IVIFS is that the values of its membership function and nonmembership function are intervals rather than exact numbers.

In this study, we introduce a fuzzy diagnostic method based on IVIFS. As an approach to medical diagnosis, we apply the method to differentiate patients according to the types of primary headache. A headache is a condition of head pain; in addition, sometimes neck or upper back pain may also be interpreted as a headache. Two categories of headaches are the primary and secondary headache. A primary headache is not associated with other diseases. Examples of primary headache are migraines, tension, and cluster headaches. A secondary headache is caused by associated diseases.

\footnotetext{
This research was supported by Basic Science Research Program through the National Research Foundation of Korea (NRF) funded by the Ministry of Education, Science and Technology (2011-0008954).

${ }^{1}$ Corresponding author: Professor, Department of Statistics (Institute of Applied Statistics), Chonbuk National University, 664-14 Dukjin-Dong, Chonbuk 561-756, Korea. E-mail: jyahn@jbnu.ac.kr
} 
For medical diagnosis of primary headache, we use an interview chart with interval fuzzy degrees based on the relation among symptoms and three types (migraines, tension, and cluster) of headache. In addition, we utilize the interval-valued intuitionistic fuzzy weighted arithmetic average(IIFWAA) operator. In the operator, we propose the occurrence of information of symptoms as weights. The stages for the medical diagnosis are as follows: First, we collect the patient's degrees and confirmability degrees. Second, we calculate IIFWAA from the symptoms of the same group (type). Lastly, we preliminarily diagnose using the max-min composition.

In Section 2 of this paper, we briefly review the basic concepts of fuzzy sets and an application of fuzzy sets in medical diagnosis. In Section 3, we introduce an interview chart to aid with the medical diagnosis of primary headaches and the IIFWAA operator to aggregate fuzzy information from the symptoms. In Section 4, we illustrate the performance of the interview chart and the operator by a numerical example. We finish with a brief discussion in Section 5.

\section{Preliminaries}

\subsection{Fuzzy sets}

Since Zadeh (1965) introduced fuzzy sets, many approaches and theories treating imprecision and uncertainty have been proposed. Some of these theories, such as IFS, IVFS, and IVIFS theory, are extensions of FS theory and others try to handle imprecision and uncertainty in different ways (Park et al., 2008).

The concept of IFS has been introduced by Atanassov and Gargov (1989) as a generalization concept of FS. Since the first public statement of this notion was made in 1983, IFS has become a popular topic of investigation in the FS community (Buckley, 2006; Seising, 2004). However, there is a current debate over the use of the name 'intuitionistic'. Debois et al. (2005) pointed out a terminological clash between Atanassov's 'intuitionistic fuzzy sets' and what is currently understood as intuitionistic logic, and argued that the name 'intuitionistic fuzzy sets' for Atanassov theory is unsuitable and misleading. We, however, use the name 'intuitionistic' in the sense of Atanassov in this study.

Definition 1. Let $X$ is a set(space), with a generic element of $X$ denoted by $x$, that is $X=\{x\}$. Then a FS is defined as follows (Zadeh, 1965):

$$
A=\left\{<x, \mu_{A}(x)>\mid x \in X\right\},
$$

where $\mu_{A}(x): X \rightarrow[0,1]$ is the membership function of the FS $A, \mu_{A}(x) \in[0,1]$ is the degree of membership of the element $x$ to the set $A$.

Definition 2. For a set X, an IFS A in the sense of Atanassov and Gargov (1989) is given by

$$
A=\left\{<x, \mu_{A}(x), v_{A}(x)>\mid x \in X\right\}
$$

where the functions $\mu_{A}: X \rightarrow[0,1]$ and $v_{A}: X \rightarrow[0,1]$, with the condition $0 \leq \mu_{A}(x)+v_{A}(x) \leq$ $1, \forall x \in X$.

The numbers, $\mu_{A}(x) \in[0,1]$ and $v_{A}(x) \in[0,1]$, denote the degree of membership and the degree of non-membership of the element $x$ to the set A, respectively. For each IFS $A$ in $X$, the amount $\pi_{A}(x)=1-\left(\mu_{A}(x)+v_{A}(x)\right)$ is called the degree of indeterminacy (hesitation part), which may cater to membership value, non-membership value or both. 
Definition 3. Let $[I]$ be the set of all closed subintervals of the interval $[0,1]$ and $M=\left[M_{L}, M_{U}\right] \in$ [I], where $M_{L}$ and $M_{U}$ are the lower extreme and the upper extreme, respectively. For a set $X$, an IVFS A is given by

$$
A=\left\{<x, M_{A}(x)>\mid x \in X\right\},
$$

where the function $M_{A}: X \rightarrow[I]$ defines the degree of membership of an element $x$ to $A$, and $M_{A}(x)=$ $\left[M_{L}(x), M_{U}(x)\right]$ is called an interval-valued fuzzy number (Zadeh, 1975).

Definition 4. For a set X, an IVIFS A is an object having the form (Atanassov and Gargov, 1989):

$$
A=\left\{<x, M_{A}(x), N_{A}(x)>\mid x \in X\right\},
$$

where $M_{A}: X \rightarrow[I]$ and $N_{A}: X \rightarrow[I]$ represent the degree of membership and non-membership, and $0 \leq \sup \left(M_{A}(x)\right)+\sup \left(N_{A}(x)\right) \leq 1, \forall x \in X$. For convenience, let $M_{A}(x)=[a, b], N_{A}(x)=[c, d]$, so $A=([a, b],[c, d])$.

\subsection{Application of fuzzy sets in medical diagnosis}

The fuzzy sets have been utilized in several different approaches to model the diagnostic process (Aversa et al., 2002; Innocent and John, 2004; Yamada, 2004). In this section, we present an application of IFS theory in Sanchez's approach two medical diagnosis (Sanchez, 1979). He represented the medical knowledge of the physician as a fuzzy relation between symptoms and diseases. The approach was elaborated by Adlassnig (1986) and applied in many studies such as Ahn et al. (2008) and Kumar et al. (2001).

Let $S=\left\{S_{1}, \ldots, S_{m}\right\}, D=\left\{D_{1}, \ldots, D_{n}\right\}$, and $P=\left\{P_{1}, \ldots, P_{q}\right\}$ denote the sets of symptoms, diseases, and patients, respectively. Two fuzzy relations, $Q$ and $R$, are defined as follows:

$$
\begin{aligned}
& Q=\left\{<(p, s), \mu_{Q}(p, s), v_{Q}(p, s)>\mid(p, s) \in P \times S\right\}, \\
& R=\left\{<(s, d), \mu_{R}(s, d), v_{R}(s, d)>\mid(s, d) \in S \times D\right\},
\end{aligned}
$$

where $\mu_{Q}(p, s)$ and $v_{Q}(p, s)$ indicate the occurrence degrees for patient's symptoms, i.e., $\mu_{Q}(p, s)$ indicates the degree to which the symptom $s$ appears in patient $p$, and $v_{Q}(p, s)$ indicates the degree to which the symptom $s$ does not appear in patient $p$. Similarly, $\mu_{R}(s, d)$ and $v_{R}(s, d)$ indicate the confirmability degrees, i.e. $\mu_{R}(s, d)$ is the degree to which symptom $s$ confirms the presence of disease $d$, and $v_{R}(s, d)$ the degree to which the symptom $s$ does not confirm the presence of disease $d$, respectively. Note that $Q$ is defined on the set $P \times S$ and $R$ on the set $S \times D$. The composition $T$ of $R$ and $Q$, $T=R \circ Q$, for the diagnosis of a disease describes the state of patients in terms of disease as a fuzzy relation from $P$ to $D$ given by the membership and non-membership functions:

$$
\begin{aligned}
\mu_{T}(p, d) & =\max _{s}\left\{\min \left[\mu_{Q}(p, s), \mu_{R}(s, d)\right]\right\}, \\
v_{T}(p, d) & =\min _{s}\left\{\max \left[v_{Q}(p, s), v_{R}(s, d)\right]\right\},
\end{aligned}
$$

for all $p \in P$ and $d \in D$.

This max-min composition is normally applied when a system involves conservative solutions in the sense that the goodness of one value cannot compensate the badness of another value (Zimmerman, $1991)$, and the diagnosis approach using the measures, $\mu_{T}(p, d)$ and $v_{T}(p, d)$, is quite a conservative method. 


\section{A Medical Diagnosis of a Headache}

\subsection{Interview chart}

In the diagnosis of a headache, the initial information (so-called symptoms) of the patient is collected through patient's medical history and interview. Therefore, a screening method that uses a questionnaire is helpful in the diagnosis of headaches and the interview chart is a leading part.

In our earlier works (Ahn et al., 2003; Kim et al., 2007), we developed an interview chart for the preliminary diagnosis of a headache, where the qualitative data from the interview chart were obtained and then quantified by dual scaling. However, the method has some problems such as loss of information and insufficient use of the physician's knowledge.

In the next study (Ahn et al., 2008), an improvement of developed interview chart has been implemented, as an extended version of the previous interview chart, based on the physician's knowledge. In the chart, we reformed the fuzzy degrees and added some composite symptoms. The chart consisted of 22,17 , and 14 symptoms for the three types of headache, respectively. The chart was investigated by 5 physicians. We estimated headache labels of patients using the information obtained from the chart. The two charts above had an exact number in $[0,1]$ as the membership/non-membership degrees.

In a recent study (Ahn et al., 2011), we developed an IVIFS version of the interview chart developed in (Ahn et al., 2008). In this study, we slightly improve the interview chart. Some degrees are corrected in the chart based on the opinion of physicians, and composite symptoms are removed. The chart consists of 20,15, and 13 symptoms for the three types of headache, respectively. The chart consists of symptoms of three groups with similar characteristics. Each item has confirmability degrees with the relation among symptoms and three types of headache, and has an interval-value in $[0,1]$ as the degrees.

\subsection{A fuzzy operator}

In diagnosis with IFS data, generally we determine the diagnostic labels of patient $p$ for any disease $d$ such that both inequalities $0.5<\mu_{T}(p, d)$ and $v_{T}(p, d)<0.5$ are satisfied. However, the diagnosis measure based on max-min composition leads to conservative results. For example, let use the confirmability membership degrees $\mu_{R}(s, d)$ for a patient are $0.7,0.4$, and 0.7 . Then the diagnosis measure $\mu_{T}(p, d)$ based on max-min composition can take 0.4 . However, if the symptoms are significantly associated with a disease, it is reasonable that the diagnosis measure has a value above 0.4 .

To complement the shortcomings of the max-min composition, we utilize the IIFWAA operator developed by $\mathrm{Xu}$ (2007). The operator is to aggregate the interval-valued intuitionistic fuzzy information corresponding to the degrees for patient's symptoms and confirmability degrees. It is defined as follows.

Definition 5. Let $A_{j}=\left(\left[a_{j}, b_{j}\right],\left[c_{j}, d_{j}\right]\right)(j=1,2, \ldots, n)$ be a collection of interval-valued intuitionistic fuzzy values. Then,

$$
\begin{aligned}
& \qquad \operatorname{IIFWAA}\left(A_{1}, A_{2}, \ldots, A_{n}\right)=\left(\left[1-\Pi_{j=1}^{n}\left(1-a_{j}\right)^{\omega_{j}}, 1-\Pi_{j=1}^{n}\left(1-b_{j}\right)^{\omega_{j}}\right],\left[\Pi_{j=1}^{n}\left(c_{j}\right)^{\omega_{j}}, \Pi_{j=1}^{n}\left(d_{j}\right)^{\omega_{j}}\right]\right), \\
& \text { where } \omega=\left(\omega_{1}, \omega_{2}, \ldots, \omega_{n}\right)^{T} \text { is the weight vector of } A_{j}, \omega_{j}>0 \text {, and } \sum_{j=1}^{n} \omega_{j}=1 .
\end{aligned}
$$

An issue in the use of the IIFWAA operator is to determine the weights. In this study, we use the occurrence proportion of symptoms of the same group as the weights, i.e., $\omega_{j}=o p_{j} / \sum_{j=1}^{n} o p_{j}$. The 
Table 1: Occurrence degrees for $P_{1}$ 's symptoms

\begin{tabular}{ccccccccccc}
\hline \hline Symptom & M5 & M8 & M18 & M19 & T3 & T5 & T10 & C4 & C12 & C13 \\
\hline$M_{Q}$ & {$[0.5,0.6]$} & {$[0.6,0.7]$} & {$[0.5,0.6]$} & {$[0.4,0.6]$} & {$[0.6,0.7]$} & {$[0.6,0.7]$} & {$[0.7,0.8]$} & {$[0.5,0.6]$} & {$[0.4,0.6]$} & {$[0.3,0.4]$} \\
$N_{Q}$ & {$[0.2,0.3]$} & {$[0.1,0.3]$} & {$[0.1,0.3]$} & {$[0.1,0.2]$} & {$[0.1,0.2]$} & {$[0.1,0.3]$} & {$[0.1,0.2]$} & {$[0.1,0.2]$} & {$[0.2,0.3]$} & {$[0.1,0.2]$} \\
Occ. prop. & 0.2 & 0.4 & 0.3 & 0.1 & 0.2 & 0.2 & 0.6 & 0.3 & 0.6 & 0.1 \\
\hline \hline
\end{tabular}

Table 2: Confirmability degrees

\begin{tabular}{|c|c|c|c|c|c|c|}
\hline \multirow{2}{*}{ Symptom } & \multicolumn{2}{|c|}{ Migraine } & \multicolumn{2}{|c|}{ Tension } & \multicolumn{2}{|c|}{ Cluster } \\
\hline & $M_{R}$ & $N_{R}$ & $M_{R}$ & $N_{R}$ & $M_{R}$ & $N_{R}$ \\
\hline M5 & {$[0.5,0.6]$} & {$[0.2,0.3]$} & {$[0.4,0.5]$} & {$[0.3,0.4]$} & {$[0.3,0.5]$} & {$[0.3,0.4]$} \\
\hline M8 & {$[0.6,0.7]$} & {$[0.1,0.2]$} & {$[0.2,0.3]$} & {$[0.5,0.6]$} & {$[0.4,0.5]$} & {$[0.4,0.5]$} \\
\hline M18 & {$[0.6,0.7]$} & {$[0.2,0.3]$} & {$[0.2,0.4]$} & {$[0.4,0.6]$} & {$[0.4,0.6]$} & {$[0.1,0.2]$} \\
\hline M19 & {$[0.5,0.6]$} & {$[0.1,0.2]$} & {$[0.1,0.2]$} & {$[0.6,0.7]$} & {$[0.3,0.4]$} & {$[0.3,0.5]$} \\
\hline $\mathrm{T} 3$ & {$[0.3,0.4]$} & {$[0.4,0.5]$} & {$[0.6,0.7]$} & {$[0.1,0.2]$} & {$[0.2,0.3]$} & {$[0.5,0.6]$} \\
\hline T5 & {$[0.3,0.4]$} & {$[0.5,0.6]$} & {$[0.5,0.7]$} & {$[0.1,0.2]$} & {$[0.1,0.3]$} & {$[0.5,0.7]$} \\
\hline $\mathrm{T} 10$ & {$[0.2,0.3]$} & {$[0.3,0.5]$} & {$[0.4,0.5]$} & {$[0.2,0.3]$} & {$[0.1,0.2]$} & {$[0.6,0.7]$} \\
\hline $\mathrm{C} 4$ & {$[0.5,0.6]$} & {$[0.2,0.3]$} & {$[0.1,0.2]$} & {$[0.6,0.7]$} & {$[0.6,0.7]$} & {$[0.1,0.2]$} \\
\hline $\mathrm{C} 12$ & {$[0.2,0.3]$} & {$[0.4,0.5]$} & {$[0.3,0.4]$} & {$[0.2,0.3]$} & {$[0.5,0.6]$} & {$[0.1,0.3]$} \\
\hline $\mathrm{C} 13$ & {$[0.3,0.4]$} & {$[0.2,0.3]$} & {$[0.2,0.3]$} & {$[0.3,0.4]$} & {$[0.4,0.5]$} & {$[0.2,0.3]$} \\
\hline
\end{tabular}

Table 3: IIFWAA occurrence degrees for $P_{1}$ 's symptoms

\begin{tabular}{cccc}
\hline \hline $\mathrm{Q}$ & Symptom M & Symptom T & Symptom C \\
\hline$P_{1}$ & $([0.53,0.64],[0.11,0.29])$ & $([0.66,0.76],[0.1,0.22])$ & $([0.42,0.58],[0.15,0.26])$ \\
\hline \hline
\end{tabular}

Table 4: IIFWAA confirmability degrees

\begin{tabular}{cccc}
\hline \hline $\mathrm{R}$ & Migraine & Tension & Cluster \\
\hline Symptom M & $([0.57,0.67],[0.14,0.24])$ & $([0.24,0.37],[0.43,0.56])$ & $([0.37,0.52],[0.24,0.36])$ \\
Symptom T & $([0.24,0.34],[0.35,0.52])$ & $([0.47,0.59],[0.15,0.26])$ & $([0.12,0.24],[0.56,0.68])$ \\
Symptom C & $([0.31,0.42],[0.30,0.41])$ & $([0.24,0.34],[0.29,0.40])$ & $([0.52,0.62],[0.11,0.27])$ \\
\hline \hline
\end{tabular}

occurrence information of symptoms is an important factor for the diagnosis of headaches, while the information is disregarded in many researches for medical diagnosis (Adlassnig, 1986; Kumar et al., 2001). In particular, it could play a significant role in diagnosis when many types of headaches are presented in patients.

The aggregation result IIFWAA with the weights is still IVIFS and is not very sensitive to $A_{j}$. For medical diagnosis, we first calculate IIFWAA from the degrees of symptoms of the same group and then use the max-min composition.

\section{Illustrative Example}

Headaches have many causes and symptoms and they may be hard to treat since the causes are so varied. Common symptoms of headaches are constant ache in the head and/or neck, fever, vomiting, lack of concentration, problems with hearing and vision, and feeling pressure behind the eyes. As there are different types of headaches, however, the symptoms of headaches are also different for each type of headache. In addition, patients may have more than one type of primary headache - for example, migraine without aura and tension type headache - and each headache type should be dealt with separately. In order to find a treatment for headaches, it is important to get a proper diagnosis of the type of headache.

In this section, we present a numerical example to illustrate medical diagnosis process. Let us consider patient $P_{1}$. $P_{1}$ 's symptoms are (M5, M8, M18, M19) of migraine, (T3, T5, T10) of 
Table 5: Composition degrees for $P_{1}$ 's diseases

\begin{tabular}{cccc}
\hline \hline $\mathrm{T}$ & Migraine & Tension & Cluster \\
\hline$P_{1}$ & $([0.53,0.64],[0.14,0.24])$ & $([0.47,0.59],[0.15,0.26])$ & $([0.42,0.58],[0.15,0.26])$ \\
\hline \hline
\end{tabular}

Table 6: Composition degrees for $P_{1}$ 's diseases (weights: $1 / n$ )

\begin{tabular}{cccc}
\hline \hline $\mathrm{T}$ & Migraine & Tension & Cluster \\
\hline$P_{1}$ & $([0.51,0.63],[0.14,0.24])$ & $([0.51,0.64],[0.13,0.23])$ & $([0.40,0.54],[0.13,0.26])$ \\
\hline \hline
\end{tabular}

tension headache, and $(\mathrm{C} 4, \mathrm{C} 12, \mathrm{C} 13)$ of cluster headache. Table 1 is the occurrence degrees, $<M_{Q}(p, s), N_{Q}(p, s)>$ assigned by a physician, for $P_{1}$ 's symptoms. Table 2 is the confirmability degrees, $<M_{R}(s, d), N_{R}(s, d)>$, indicated in the interview chart.

First, based on Table 1 and Table 2, Table 3 and Table 4 are calculated by applying IIFWAA operator of the Definition 5. Table 3 is IIFWAA $<M_{Q}(p, s), N_{Q}(p, s)>$, and Table 4 is IIFWAA $<M_{R}(s, d), N_{R}(s, d)>$. For example, [0.57, 0.67], an IIFWAA $M_{R}(s, d)$ of Table 4, is calculated as follows: From the confirmability membership degrees ([0.5, 0.6], [0.6, 0.7], [0.6, 0.7], [0.5, 0.6]) of the symptoms (M5, M8, M18, M19) and $\omega=(0.2,0.4,0.3,0.1)$,

$$
\begin{aligned}
& 0.57=1-\left\{(1-0.5)^{0.2}\right\} *\left\{(1-0.6)^{0.4}\right\} *\left\{(1-0.6)^{0.3}\right\} *\left\{(1-0.5)^{0.1}\right\} \\
& 0.67=1-\left\{(1-0.6)^{0.2}\right\} *\left\{(1-0.7)^{0.4}\right\} *\left\{(1-0.7)^{0.3}\right\} *\left\{(1-0.6)^{0.1}\right\} .
\end{aligned}
$$

An IIFWAA $N_{R}(s, d)$ of Table $4,[0.14,0.24]$, is calculated as follows: From the confirmability non-membership degrees $([0.2,0.3],[0.1,0.2],[0.2,0.3],[0.1,0.2])$ of the symptoms (M5, M8, M18, M19) and $\omega=(0.2,0.4,0.3,0.1)$,

$$
\begin{aligned}
& 0.14=\left\{0.2^{0.2}\right\} *\left\{0.1^{0.4}\right\} *\left\{0.2^{0.3}\right\} *\left\{0.1^{0.1}\right\}, \\
& 0.24=\left\{0.3^{0.2}\right\} *\left\{0.2^{0.4}\right\} *\left\{0.3^{0.3}\right\} *\left\{0.2^{0.1}\right\} .
\end{aligned}
$$

Second, we can obtain Table 5 applying max-min composition in Table 3 and Table 4. Table 5 is the composition degrees, $<M_{T}\left(P_{1}, d\right), N_{T}\left(P_{1}, d\right)>$, for $P_{1}$ 's diseases. As a result, we can diagnose that patient $P_{1}$ suffers from migraine with $58.5 \%$ (average of lower and upper extreme) degree of membership, tension headache with about $53 \%$ degree, and cluster headache with about $50 \%$ degree.

However, if we use $\omega_{j}=(1 / n, 1 / n, \ldots, 1 / n)$ in this example, we will diagnose that patient $P_{1}$ suffers from migraine with $57 \%$ degree of membership, tension headache with about $57.5 \%$ degree, and no cluster headache (see Table 6). In addition, if we apply only max-min composition without the use of IIFWAA operator, we will diagnose that patient $P_{1}$ suffers from migraine with $50 \%$ degree of membership.

\section{Conclusion}

IVIFS, a generalization of IVFS, has received more attention in many fields since previously invented. In this paper, IVIFS theory has been applied to make a diagnosis of headaches, as a new approach on decision support practices in medicine. To apply a fuzzy relation in medical diagnosis, we developed an interval-valued interview chart with an interval fuzzy degree assigned to the relation among symptoms and three types of headaches, and utilized the IIFWAA operator to calculate fuzzy degrees from the symptoms. We first calculate IIFWAA and then use the max-min composition. The result of the example indicates that it is possible to classify headache using our diagnosis method. The method has been used experimentally in the neurology field to support a physician's decision. 


\section{References}

Adlassnig, K. P. (1986). Fuzzy set theory in medical diagnosis, IEEE Transactions on Systems, Man, and Cybernetics, 16, 260-265.

Ahn, J. Y., Han, K. S., Oh, S. Y. and Lee, C. D. (2011). An application of interval-valued intuitionistic fuzzy sets for medical diagnosis of headache, International Journal of Innovative Computing, Information and Control, 7, 2755-2762.

Ahn, J. Y., Kim, Y. H. and Kim, S. K. (2003). A fuzzy differential diagnosis of headache applying linear regression method and fuzzy classification, IEICE Transactions on Information and Systems, E86-D, 2790-2793.

Ahn, J. Y., Mun, K. S., Kim, Y. H., Oh, S. Y. and Han, B. S. (2008). A fuzzy method for medical diagnosis of headache, IEICE Transactions on Information and Systems, E91-D, 1215-1217.

Atanassov, K. (1986). Intuitionistic fuzzy sets, Fuzzy Sets and Systems, 20, 87-96.

Atanassov, K. and Gargov, G. (1989). Interval-valued intuitionistic fuzzy sets, Fuzzy Sets and Systems, 31, 343-349.

Aversa, F., Gronda, E., Pizzuti, S. and Aragno, C. (2002). A fuzzy logic approach to decision support in medicine, Proceedings of the Conference on Systemics, Cybernetics and Informatics.

Buckley, J. J. (2006). Fuzzy Probability and Statistics, Springer.

Dubois, D., Gottwald, S., Hajek, P., Kacprzyk, J. and Prade, H. (2005). Terminological difficulties in fuzzy set theory - The case of Intuitionistic Fuzzy Sets, Fuzzy Sets and Systems, 156, 485-491.

Innocent, P. R. and John, R. I. (2004). Computer aided fuzzy medical diagnosis, Information Sciences, 162, 81-104.

Kim, Y. H., Kim, S. K., Oh, S. Y. and Ahn, J. Y. (2007). A fuzzy differential diagnosis of headache, Journal of the Korean Data and Information Science Society, 18, 429-438.

Kumar, S., Biswas, R. and Roy, A. R. (2001). An application of intuitionistic fuzzy sets in medical diagnosis, Fuzzy Sets and Systems, 117, 209-213.

Park, J. H., Lim, K. M., Park, J. S. and Kwun, Y. C. (2008). Distances between interval-valued intuitionistic fuzzy sets, Journal of Physics: Conference Series, 96.

Sanchez, E. (1979). Medical diagnosis and composite fuzzy relations, Gupta, M.M., Ragade, R.K., Yager R.R. (Eds.) Advances in Fuzzy Set Theory and Applications, 437-444.

Seising, R. (2004). A history of medical diagnosis using fuzzy relations, Proceedings in the Conference on Fuzziness.

Turksen, B. (1986). Interval valued fuzzy sets based on normal forms, Fuzzy Sets and Systems, 20, 191-210.

Xu, Z. S. (2007). Methods for aggregating interval-valued intuitionistic fuzzy information and their application to decision making, Control and Decision, 22, 215-219.

Yamada, K. (2004). Diagnosis under compound effects and multiple causes by means of the conditional causal possibility approach, Fuzzy Sets and Systems, 145, 183-212.

Zadeh, L. A. (1965). Fuzzy sets, Information and Control, 8, 338-353.

Zadeh, L. A. (1969). Biological applications of the theory of fuzzy sets and systems, Proceedings of an International Symposium on Biocybernetics of the Central Nervous System, 99-206.

Zadeh, L. A. (1975). The concept of a linguistic variable and its application to approximate reasoning, Part I, Information Science, 8, 199-249.

Zimmerman, H. J. (1991). Fuzzy Set Theory and its Application, Kluwer Academic Publishers. 